Leszek Olszowy* and Tomasz Zając

\title{
Some inequalities and superposition operator in the space of regulated functions
}

https://doi.org/10.1515/anona-2020-0050

Received August 6, 2019; accepted October 2, 2019.

Abstract: Some inequalities connected to measures of noncompactness in the space of regulated function $R(J, E)$ were proved in the paper. The inequalities are analogous of well known estimations for Hausdorff measure and the space of continuous functions. Moreover two sufficient and necessary conditions that superposition operator (Nemytskii operator) can act from $R(J, E)$ into $R(J, E)$ are presented. Additionally, sufficient and necessary conditions that superposition operator $F_{f}: R(J, E) \rightarrow R(J, E)$ was compact are given.

Keywords: space of regulated functions, measure of noncompactness, integral inequalities, superposition operator

MSC 2010: Primary 47H30, Secondary 46E40

\section{Introduction}

When studying solvability of various non-linear equations, it is significant to properly choose the space in which the equation is considered. Knowledge about some properties of the space e.g. easy to calculate formulas for measures of noncompactness or characteristic of superposition operator etc. combined with fixed point theorems allow to obtain general conditions for solvability of studied equations.

The space of regulated functions $R(J, E)$, where $J=[a, b] \subset \mathbb{R}$ and $E$ is a Banach space, is one of such spaces, recently intensively studied (see [1-14]). So far except stating general properties of this space [1, 5, 7-12] it is also possible to use formulas for measures of noncompactness, conditions sufficient for the superposition operator $F_{f}$ to act from $R(J, E)$ into $R(J, E)$, and conditions for continuity of this operator [2-6, 12-13]. However, so far non-trivial sufficient and necessary conditions for compactness of the superposition operator $F_{f}: R(J, E) \rightarrow R(J, E)$ were not known. There was also lack of any estimations of such measures, so often needed. This paper will try to fill these gaps.

In the third chapter several theorems dealing with various types of inequalities, integral, but not only integral, that hold in the spaces of regulated functions and are expressed in terms of measures of noncompactness, will be formulated. These inequalities are analogues of known and often used inequalities holding in the class of continuous functions. In the fourth chapter two theorems (Theorem 4.4 and Theorem 4.6) that give sufficient and necessary conditions that superposition operator acted from the space of regulated functions into that space, will be presented. Known so far results in this area usually give only sufficient conditions and the only known sufficient and necessary conditions are actually rather "tautological". Moreover, sufficient and necessary conditions that superposition operator is compact in the space of regulated functions will be given (Theorem 4.9).

*Corresponding Author: Leszek Olszowy, Department of Nonlinear Analysis, Rzeszów University of Technology, Al. Powstańców Warszawy 8, 35-959, Rzeszów, Poland, E-mail: lolszowy@prz.edu.pl

Tomasz Zając, Department of Nonlinear Analysis, Rzeszów University of Technology, Al. Powstańców Warszawy 8, 35-959, Rzeszów, Poland, E-mail: tzajac@prz.edu.pl 


\section{Notation, definitions and auxiliary facts}

This section is focuses on recalling some facts which will be used in our investigations.

Assume that $E$ is a real Banach space with the norm $\|\cdot\|$ and the zero element $\theta$. Denote by $B_{E}(x, r)$ the closed ball centered at $x$ and with radius $r$. The ball $B_{E}(\theta, r)$ will be denoted by $B_{E}(r)$. We write $\bar{X}$, $\operatorname{Conv} X$ to denote the closure and the convex closure of a set $X$, respectively. The symbol $\|X\|$ will stand for the norm of the set $X \subset E$ i.e., we have $\|X\|:=\sup \{\|x\|: x \in X\}$. Furthermore, let $\mathfrak{M}_{E}$ denote the family of all nonempty and bounded subsets of $E$ and $\mathfrak{N}_{E}$ its subfamily consisting of all relatively compact sets. We accept the following definition of a measure of noncompactness [15].

Definition 2.1. A mapping $\mu: \mathfrak{M}_{E} \rightarrow \mathbb{R}_{+}=[0, \infty)$ is said to be a measure of noncompactness in $E$ if it satisfies the following conditions:

$1^{o}$ The family ker $\mu:=\left\{X \in \mathfrak{M}_{E}: \mu(X)=0\right\}$ is nonempty and ker $\mu \subset \mathfrak{N}_{E}$.

$2^{o} \quad X \subset Y \Rightarrow \mu(X) \leq \mu(Y)$.

$3^{\circ} \mu(\operatorname{Conv} X)=\mu(X)$.

$4^{o} \mu(\lambda X+(1-\lambda) Y) \leq \lambda \mu(X)+(1-\lambda) \mu(Y)$ for $\lambda \in[0,1]$.

$5^{o}$ If $\left(X_{n}\right)$ is a sequence of closed sets from $\mathfrak{M}_{E}$ such that $X_{n+1} \subset X_{n}(n=1,2, \ldots)$ and if $\lim _{n \rightarrow \infty} \mu\left(X_{n}\right)=0$, then the intersection $X_{\infty}:=\bigcap_{n=1}^{\infty} X_{n}$ is nonempty.

Subsequently, we will use measures of noncompactness having some additional properties. Namely, a measure $\mu$ is said to be sublinear if it satisfies the following two conditions:

$6^{0} \quad \mu(\lambda X)=|\lambda| \mu(X), \lambda \in \mathbb{R}$.

$7^{\circ} \mu(X+Y) \leq \mu(X)+\mu(Y)$.

A sublinear measure of noncompactness $\mu$ satisfying the condition (strong maximum property)

$8^{o} \quad \mu(X \cup Y)=\max \{\mu(X), \mu(Y)\}$

and such that $\operatorname{ker} \mu=\mathfrak{N}_{E}$ is said to be regular.

Except condition $8^{\circ}$ we can also consider the condition (weak maximum property)

$9^{\circ} \quad \mu(X \cup\{y\})=\mu(X), y \in E$.

For a given nonempty bounded subset $X$ of $E$, we denote by $\beta_{E}(X)$ the so-called Hausdorff measure of noncompactness of $X$. This quantity is defined by formula

$$
\beta_{E}(X):=\{r>0: X \text { has a finite } r \text {-net in } E\} .
$$

The function $\beta_{E}$ is an example of regular measure of noncompactness in $E$.

Now we recall some facts concerning regulated functions.

Definition 2.2. A function $x:[a, b] \rightarrow E$, where $E$ is a topological vector space, is said to be a regulated function if for every $t \in[a, b)$ the right-sided limit $x\left(t^{+}\right):=\lim _{s \rightarrow t^{+}} x(s)$ exists and for every $t \in(a, b]$ the leftsided limit $x\left(t^{-}\right):=\lim _{s \rightarrow t^{-}} x(s)$ exists.

From now on, real Banach space will be denoted by $E$.

Denote by $R(J, E)$ the space consisting of all regulated functions defined on the interval $J=[a, b]$ with values in a real Banach space $E$. Since every regulated function $x \in R(J, E)$ is bounded on the interval $J$, then the space $R(J, E)$ can be normed via the classical supremum norm

$$
\|x\|_{\infty}:=\sup \{\|x(t)\|: t \in J\} .
$$

It is easy to show that $R(J, E)$ is a real Banach space. Moreover, every regulated function $x: J \rightarrow E$ is Riemann integrable. 
Now, we remind a criterion for relative compactness in the space $R(J, E)$. To this end, we introduce the concept of a equiregulated subset of the space $R(J, E)$ (cf. [3, 9]).

Definition 2.3. We will say that the set $X \subset R(J, E)$ is equiregulated on the interval $J$ if the following two conditions are satisfied:

$$
\begin{aligned}
& \forall_{t \in(a, b]} \forall_{\varepsilon>0} \exists_{\delta>0} \forall_{x \in X} \forall_{t_{1}, t_{2} \in(t-\delta, t) \cap[a, b]}\left\|x\left(t_{2}\right)-x\left(t_{1}\right)\right\| \leq \varepsilon, \\
& \forall_{t \in[a, b)} \forall_{\varepsilon>0} \exists_{\delta>0} \forall_{x \in X} \forall_{t_{1}, t_{2} \in(t, t+\delta) \cap[a, b]}\left\|x\left(t_{2}\right)-x\left(t_{1}\right)\right\| \leq \varepsilon .
\end{aligned}
$$

Theorem 2.4. [3-5, 9] A nonempty subset $X \subset R(J, E)$ is relatively compact in $R(J, E)$ if and only if $X$ is equiregulated on the interval $J$ and the sets $X(t)$ are relatively compact in $E$ for $t \in J$.

Now we are going to recall the construction of a measure of noncompactness in the space $R(J, E)$. To this end, let us take a set $X \in \mathfrak{M}_{R(J, E)}$. For $x \in X$ and $\varepsilon>0$ let us denote the following quantities:

$$
\begin{array}{ll}
\omega^{-}(x, t, \varepsilon):=\sup \left\{\left\|x\left(t_{2}\right)-x\left(t_{1}\right)\right\|: t_{1}, t_{2} \in(t-\varepsilon, t) \cap J\right\}, & t \in(a, b], \\
\omega^{+}(x, t, \varepsilon):=\sup \left\{\left\|x\left(t_{2}\right)-x\left(t_{1}\right)\right\|: t_{1}, t_{2} \in(t, t+\varepsilon) \cap J\right\}, & t \in[a, b) .
\end{array}
$$

The quantities $\omega^{-}(x, t, \varepsilon)$ and $\omega^{+}(x, t, \varepsilon)$ can be interpreted as left hand and right hand sided moduli of convergence of the function $x$ at the point $t$. Furthermore, let us put:

$$
\begin{gathered}
\omega^{-}(X, t, \varepsilon):=\sup \left\{\omega^{-}(x, t, \varepsilon): x \in X\right\}, \quad t \in(a, b], \\
\omega^{+}(X, t, \varepsilon):=\sup \left\{\omega^{+}(X, t, \varepsilon): x \in X\right\}, \quad t \in[a, b), \\
\omega^{-}(X, t):=\lim _{\varepsilon \rightarrow 0^{+}} \omega^{-}(X, t, \varepsilon), \quad t \in(a, b], \\
\omega^{+}(X, t):=\lim _{\varepsilon \rightarrow 0^{+}} \omega^{+}(X, t, \varepsilon), \quad t \in[a, b), \\
\omega^{-}(X):=\sup _{t \in(a, b]} \omega^{-}(X, t), \quad \omega^{+}(X):=\sup _{t \in[a, b)} \omega^{+}(X, t) .
\end{gathered}
$$

Finally, let us define the following quantity

$$
\mu_{m}(X):=\max \left\{\omega^{-}(X), \omega^{+}(X)\right\}+\sup _{t \in J} \beta_{E}(X(t)) .
$$

Theorem 2.5. [13] The function $\mu_{m}$ given by formula (2.1) satisfies conditions $1^{\circ}-7^{\circ}$ and $9^{\circ}$ in the space $R(J, E)$.

Remark 2.6. Above construction of the measure (2.1) addresses inaccuracies existent in the construction of measures given in $[3,4]$.

\section{Inequalities including measures of noncompactness}

This section we start with the proof of inequality which analogue for equicontinuous family of continuous functions is often used in studying solvability of nonlinear equations.

For a fixed nonempty subset $X \subset R(J, E)$, let us put

$$
\begin{gathered}
X(t):=\{x(t): x \in X\}, \quad t \in J, \\
\int_{a}^{b} X(t) d t:=\left\{\int_{a}^{b} x(t) d t: x \in X\right\} .
\end{gathered}
$$


Theorem 3.1. Let $X \subset R(J, E)$ be nonempty, bounded and equiregulated. Then the function $J \ni t \mapsto \beta_{E}(X(t)) \in$ $\mathbb{R}_{+}$is regulated and the following inequality holds

$$
\beta_{E}\left(\int_{a}^{b} X(t) d t\right) \leq \int_{a}^{b} \beta_{E}(X(t)) d t
$$

Proof. Let us fix $\varepsilon>0$. The condition of $X$ being equiregulated implies that

$$
\begin{aligned}
& \forall_{s \in[a, b)} \exists_{\delta_{s}>0} \quad \omega^{+}\left(X, s, \delta_{s}\right)<\varepsilon \text { and }\left(s, s+\delta_{s}\right) \subset[a, b), \\
& \forall_{s \in(a, b]}{ }_{\delta_{s}^{\prime}>0} \quad \omega^{-}\left(X, s, \delta_{s}^{\prime}\right)<\varepsilon \text { and }\left(s-\delta_{s}^{\prime}, s\right) \subset(a, b] .
\end{aligned}
$$

Since the family of intervals $\left\{\left[a, a+\delta_{a}\right),\left(b-\delta_{b}^{\prime}, b\right],\left(s-\delta_{s}^{\prime}, s+\delta_{s}\right): s \in(a, b)\right\}$ is an open cover of compact interval $J$, then there is a finite subcover. Obviously it contains intervals $\left[a, a+\delta_{a}\right)$ and $\left(b-\delta_{b}^{\prime}, b\right]$. From this finite subcover we can choose following subcover

$$
\begin{gathered}
{\left[s_{0}, s_{0}+\delta_{s_{0}}\right),\left(s_{1}-\delta_{s_{1}}^{\prime}, s_{1}+\delta_{s_{1}}\right),\left(s_{2}-\delta_{s_{2}}^{\prime}, s_{2}+\delta_{s_{2}}\right), \ldots,} \\
\ldots,\left(s_{k-1}-\delta_{s_{k-1}}^{\prime}, s_{k-1}+\delta_{s_{k-1}}\right),\left(s_{k}-\delta_{s_{k}}^{\prime}, s_{k}\right]
\end{gathered}
$$

such that $a=s_{0}<s_{1}<\ldots<s_{k}=b$ and additionally every two consecutive intervals from (3.3) have nonempty intersection. Now we choose one point $s_{i}^{\prime}$ belonging to each of those $k$ intersections, i.e. we have the sequence of points

$$
a=s_{0}<s_{1}^{\prime}<s_{1}<s_{2}^{\prime}<s_{2}<\ldots<s_{k-1}<s_{k}^{\prime}<s_{k}=b .
$$

For simplicity let us denote them by $t_{i}, i=0, \ldots, n$ where $n=2 k$ i.e.

$$
t_{0}=s_{0}, t_{1}=s_{1}^{\prime}, t_{2}=s_{1}, t_{3}=s_{2}^{\prime}, t_{4}=s_{2}, \ldots, t_{n-1}=s_{k}^{\prime}, t_{n}=s_{k} .
$$

From (3.1) and (3.2) we yield that

$$
\forall_{\tau_{1}, \tau_{2} \in\left(t_{i-1}, t_{i}\right)} \forall_{x \in X}\left\|x\left(\tau_{2}\right)-x\left(\tau_{1}\right)\right\| \leq \varepsilon, \quad i=1, \ldots, n .
$$

Hence

$$
\forall_{\tau_{1}, \tau_{2} \in\left(t_{i-1}, t_{i}\right)}\left|\beta_{E}\left(X\left(\tau_{2}\right)\right)-\beta_{E}\left(X\left(\tau_{1}\right)\right)\right| \leq \varepsilon, \quad i=1, \ldots, n,
$$

which means that the function $J \ni t \mapsto \beta_{E}(X(t))$ is regulated, thus Riemann integrable. Let us choose arbitrary $s_{i} \in\left(t_{i-1}, t_{i}\right), i=1, \ldots, n$. Then by (3.5) we have

$$
\left|\int_{a}^{b} \beta_{E}(X(t)) d t-\sum_{i=1}^{n}\left(t_{i}-t_{i-1}\right) \beta_{E}\left(X\left(s_{i}\right)\right)\right| \leq \sum_{i=1}^{n}\left(t_{i}-t_{i-1}\right) \varepsilon=(b-a) \varepsilon .
$$

For each $i=1, \ldots, n$ there exist $v_{j}^{i} \in E, j=1, \ldots, m_{i}$, such that

$$
X\left(s_{i}\right) \subset \bigcup_{j=1}^{m_{i}} B\left(v_{j}^{i}, \beta_{E}\left(X\left(s_{i}\right)\right)+\varepsilon\right) .
$$

Hence and by (3.4) we have

$$
\forall_{t \in\left(t_{i-1}, t_{i}\right)} X(t) \subset \bigcup_{j=1}^{m_{i}} B\left(v_{j}^{i}, \beta_{E}\left(X\left(s_{i}\right)\right)+2 \varepsilon\right), \quad i=1, \ldots, n .
$$

Let us set functions $y_{j_{1}, \ldots, j_{n}} \in R(J, E), j_{i} \in\left\{1, \ldots, m_{i}\right\}, i=1, \ldots, n$ by formulas

$$
y_{j_{1}, \ldots, j_{n}}(t):=\left\{\begin{array}{cl}
\theta & \text { for } t=t_{i}, i=0,1, \ldots n, \\
v_{j_{i}}^{i} & \text { for } t \in\left(t_{i-1}, t_{i}\right), i=1, \ldots, n .
\end{array}\right.
$$


We prove that the set of vectors given by $\int_{a}^{b} y_{j_{1}, \ldots, j_{n}}(t) d t$ for $j_{i} \in\left\{1, \ldots, m_{i}\right\}, i=1, \ldots, n$ is $\left(\int_{a}^{b} \beta_{E}(X(t)) d t+\right.$ $3 \varepsilon(b-a))$-net for $\int_{a}^{b} X(t) d t$. Let $x \in X$. Because of (3.7) there exists such a sequence $j_{1}, \ldots, j_{n}$, that

$$
\left\|x(t)-v_{j_{i}}^{i}\right\| \leq \beta_{E}\left(X\left(s_{i}\right)\right)+2 \varepsilon, \quad t \in\left(t_{i-1}, t_{i}\right), i=1, \ldots, n .
$$

Then, using (3.8) and (3.6) we have

$$
\begin{gathered}
\left\|\int_{a}^{b} x(t) d t-\int_{a}^{b} y_{j_{1}, \ldots, j_{n}}(t) d t\right\| \leq \sum_{i=1}^{n} \int_{t_{i-1}}^{t_{i}}\left\|x(t)-y_{j_{1}, \ldots, j_{n}}(t)\right\| d t \\
=\sum_{i=1}^{n} \int_{t_{i-1}}^{t_{i}}\left\|x(t)-v_{j_{i}}^{i}\right\| d t \leq \sum_{i=1}^{n}\left(t_{i}-t_{i-1}\right)\left(\beta_{E}\left(X\left(s_{i}\right)\right)+2 \varepsilon\right) \\
\leq \sum_{i=1}^{n}\left(t_{i}-t_{i-1}\right) \beta_{E}\left(X\left(s_{i}\right)\right)+2 \varepsilon(b-a) \leq \int_{a}^{b} \beta_{E}(X(t)) d t+3 \varepsilon(b-a),
\end{gathered}
$$

which means that

$$
\beta_{E}\left(\int_{a}^{b} X(t) d t\right) \leq \int_{a}^{b} \beta_{E}(X(t)) d t+3 \varepsilon(b-a),
$$

which for $\varepsilon \rightarrow 0$ proves the theorem.

Without the assumption about $X \subset R(J, E)$ being equiregulated, the function $J \ni t \mapsto \beta_{E}(X(t))$ does not have to be measurable in Lebesgue sense. However, for countable subsets of the space $R(J, E)$ we have (see [16]).

Theorem 3.2. If $\left\{x_{n}: n \in \mathbb{N}\right\} \subset R(J, E)$ and there exists Lebesgue integrable function $g: J \rightarrow \mathbb{R}_{+}$such that $\left\|x_{n}(t)\right\| \leq g(t)$ for $t \in J, n \in \mathbb{N}$, then the function $J \ni t \mapsto \beta_{E}\left(\left\{x_{n}(t): n \in \mathbb{N}\right\}\right)$ is Lebesgue integrable on $J$ and

$$
\beta_{E}\left(\left\{\int_{a}^{b} x_{n}(t) d t: n \in \mathbb{N}\right\}\right) \leq 2 \int_{a}^{b} \beta_{E}\left(\left\{x_{n}(t): n \in \mathbb{N}\right\}\right) d t .
$$

Remark 3.3. Above theorem is also true given weaker assumption that functions $x_{n}$ are strongly measurable [16]. The example from [17] shows that factor 2 from the above theorem cannot be replaced by smaller even for the sequence $\left\{x_{n}\right\}$ of regulated functions.

In some applications of measures of noncompactness the following lemma can be useful.

Lemma 3.4. [18] If $E$ is a Banach then for each non-empty and bounded set $X \subset E$ there exists such countable set $X_{0} \subset X$, that $\beta_{E}(X) \leq 2 \beta_{E}\left(X_{0}\right)$.

One can ask what can be an analogue of this lemma for measure $\mu_{m}$ and space $R(J, E)$ ? The answer is given in two following theorems.

Theorem 3.5. For each non-empty and bounded set $X \subset R(J, E)$, where $E$ is a finite dimensional Banach space, there exists such countable set $X_{0} \subset X$ that $\mu_{m}\left(X_{0}\right)=\mu_{m}(X)$.

Proof. Without loss of generality we can assume that $\max \left\{\omega^{-}(X), \omega^{+}(X)\right\}=\omega^{+}(X)$. Let $\left\{t_{n}\right\} \subset J$ be such a sequence that $\omega^{+}(X)=\sup _{n \in \mathbb{N}} \omega^{+}\left(X, t_{n}\right)$. Let us arbitrarily fix $n \in \mathbb{N}$. Then for each $i \in \mathbb{N}$ there exists such a function $x_{i}^{n} \in X$ that $\lim _{i \rightarrow \infty} \omega^{+}\left(x_{i}^{n}, t_{n}, \frac{1}{i}\right)=\omega^{+}\left(X, t_{n}\right)$. Denote $X_{0}:=\left\{x_{i}^{n}: i, n \in \mathbb{N}\right\}$. Then based on the above we have $\omega^{+}\left(X_{0}\right)=\omega^{+}(X), \omega^{-}\left(X_{0}\right) \leq \omega^{-}(X)$ and hence $\mu_{m}\left(X_{0}\right)=\mu_{m}(X)$. 
In the general case the assertion of the previous theorem has to be weakened.

Theorem 3.6. For each non-empty and bounded set $X \subset R(J, E)$ and any $\varepsilon>0$, there exists such countable set $X_{0} \subset X$, that $\mu_{m}(X) \leq 2 \mu_{m}\left(X_{0}\right)+\varepsilon$. In the above estimation factor 2 cannot be replaced by smaller (see Example 3.7).

Proof. Let $\tilde{X}_{0}$ be a countable set constructed the same way as in the proof of Theorem 3.5. Thus

$$
\max \left\{\omega^{-}(X), \omega^{+}(X)\right\}=\max \left\{\omega^{-}\left(\tilde{X}_{0}\right), \omega^{+}\left(\tilde{X}_{0}\right)\right\} .
$$

Let us arbitrarily fix $\varepsilon>0$. There exists such a number $t_{0} \in J$ that $\sup _{t \in J} \beta_{E}(X(t))<\beta_{E}\left(X\left(t_{0}\right)\right)+\varepsilon$. Using Lemma 3.4 we get that there exists such a sequence $\left\{x_{n}\right\} \subset X$ that

$$
\beta_{E}\left(X\left(t_{0}\right)\right) \leq 2 \beta_{E}\left(\left\{x_{n}\left(t_{0}\right): n \in \mathbb{N}\right\}\right) .
$$

Hence

$$
\begin{gathered}
\sup _{t \in J} \beta_{E}(X(t)) \leq 2 \beta_{E}\left(\left\{x_{n}\left(t_{0}\right): n \in \mathbb{N}\right\}\right)+\varepsilon \\
\leq 2 \sup _{t \in J} \beta_{E}\left(\left\{x_{n}(t): n \in \mathbb{N}\right\}\right)+\varepsilon .
\end{gathered}
$$

Let $X_{0}:=\tilde{X}_{0} \cup\left\{x_{n}: n \in \mathbb{N}\right\}$. Obviously $X_{0}$ is countable and

$$
\begin{gathered}
\sup _{t \in J} \beta_{E}(X(t)) \leq 2 \sup _{t \in J} \beta_{E}\left(X_{0}(t)\right)+\varepsilon, \\
\max \left\{\omega^{-}(X), \omega^{+}(X)\right\}=\max \left\{\omega^{-}\left(X_{0}\right), \omega^{+}\left(X_{0}\right)\right\} .
\end{gathered}
$$

Hence

$$
\begin{gathered}
\mu_{m}(X)=\sup _{t \in J} \beta_{E}(X(t))+\max \left\{\omega^{-}(X), \omega^{+}(X)\right\} \\
\left.\leq 2 \sup _{t \in J} \beta_{E}\left(X_{0}\right)(t)\right)+\max \left\{\omega^{-}\left(X_{0}\right), \omega^{+}\left(X_{0}\right)\right\}+\varepsilon \leq 2 \mu_{m}\left(X_{0}\right)+\varepsilon .
\end{gathered}
$$

By $\mathbf{1}_{A}$ we denote the indicator function of a subset $A$. When the subset $A=\{a\}$ is singleton, we will write $\mathbf{1}_{a}$. Additionally, for arbitrary $u \in E$ let $\widehat{u}$ denotes the function $\widehat{u}: J \rightarrow E$ given by

$$
\widehat{u}(t) \equiv u, t \in J .
$$

Example 3.7. Let $E$ be a space consisting of all bounded functions $x: \mathbb{R}_{+} \rightarrow \mathbb{R}$ such that for each of them there exists such countable set $T_{X} \subset \mathbb{R}_{+}$, that function $x(t)$ tends to 0 as $t \rightarrow \infty$ and $t \in \mathbb{R}_{+} \backslash T_{x}$. We assume the supremum norm in $E$. Now we can define a subset $A \subset E$ by $A:=\left\{\mathbf{1}_{t}: t \in \mathbb{R}_{+}\right\}$and next we define a subset $X \subset R(J, E)$ by $X:=\{\widehat{a}: a \in A\}$ where $\widehat{a}$ is defined in (3.9). Thus $\omega^{-}(X)=\omega^{+}(X)=0, \sup _{t \in J} \beta_{E}(X(t))=\beta_{E}(A)=1$ which means that $\mu_{m}(X)=1$ and for any countable subset $X_{0}=\left\{\widehat{\mathbf{1}_{t_{n}}}: n=1,2, \ldots\right\} \subset X$ we have $\omega^{-}\left(X_{0}\right)=$ $\omega^{+}\left(X_{0}\right)=0, \sup _{t \in J} \beta_{E}\left(X_{0}(t)\right)=\beta_{E}\left(\left\{\mathbf{1}_{t_{n}}: n=1,2, \ldots\right\}\right)=\frac{1}{2}$ because $\left\{\mathbf{1}_{t_{n}}: n=1,2, \ldots\right\} \subset B_{E}\left(\frac{1}{2} \sum_{n=1}^{\infty} \mathbf{1}_{t_{n}}, \frac{1}{2}\right)$ which means that $\mu_{m}\left(X_{0}\right)=\frac{1}{2}$ and that proves that factor 2 from the above theorem cannot be replaced by smaller.

\section{Superposition operator}

Consider a function $f: J \times E \rightarrow E$. Then, to every function $x: J \rightarrow E$, we may assign the function $\left(F_{f} x\right)(t):=f(t, x(t)), t \in J$. Operator $F_{f}$ defined in such way is said to be superposition (or Nemytskii) operator generated by the function $f$ (see [19-21]). In connection with the space $R(J, E)$, the natural question appears: what properties must the function $f$ satisfy in order for operator $F_{f}$ to map the space $R(J, E)$ into itself?

In the paper by Aziz [2] and Michalak [12] the following results were obtained. 
Theorem 4.1. [12] A superposition operator $F_{f}$ maps $R(J, E)$ into itself if and only if the function $f$ has the following properties:

(1) the limit $\lim _{[a, t) \times E \ni(s, v) \rightarrow(t, x)} f(s, v)$ exists for every $(t, x) \in(a, b] \times E$,

(2) the limit $\lim _{(t, b] \times E \ni(s, v) \rightarrow(t, x)} f(s, v)$ exists for every $(t, x) \in[a, b) \times E$.

Given the notation

$$
g_{t}(x):=\lim _{(t, b] \times E \ni(s, v) \rightarrow(t, x)} f(s, v), \quad t \in[a, b), x \in E,
$$

condition (2) of Theorem 4.1 can be written in an equivalent form using quantifiers

$$
\forall_{x \in E} \forall_{t \in[a, b)} \exists_{g_{t}(x) \in E} \forall_{\varepsilon>0} \exists_{\delta>0} \exists_{\tau>0} \forall_{v \in B_{E}(x, \delta)} \forall_{s \in(t, t+\tau)}\left\|g_{t}(x)-f(s, v)\right\| \leq \varepsilon .
$$

Analogically condition (1) of Theorem 4.1 can be written - we omit the details.

Theorem 4.2. [2] Suppose that the function $f(\cdot, u)$ is regulated on $[0,1]$ for all $u \in E$, and the function $f(t, \cdot)$ is continuous on $E$, uniformly with respect to $t \in J$. Then the superposition operator $F_{f}$ maps $R(J, E)$ into itself and is (norm) bounded.

Theorem 4.3. [12] A superposition operator $F_{f}$ maps $R(J, E)$ into itself is continuous if and only if a function $\tilde{f}: E \ni x \mapsto f(\cdot, x) \in R(J, E)$ is continuous.

Let us denote by $E^{E}$ the linear space consisted of all, not necessarily continuous functions $h: E \rightarrow E$. This space will become a linear topological space when we introduce a topology of almost uniform convergence through a family of pseudonorms $\left\{\|\cdot\|_{K}\right\}_{K \in \mathcal{K}}$ given by $\|h\|_{K}:=\sup _{x \in K}\|h(x)\|$ for $h \in E^{E}$, where $\mathcal{K}$ is is a family of all non-empty compact sets in $E$. So given family $\left\{h_{s}\right\}_{s \in J} \subset E^{E}$ the convergence $\left\{h_{s}\right\}$ to $h \in E^{E}$ in this topology with $s \rightarrow t \in J$ means uniform convergence $h_{s}$ to $h$ on all compact subsets in $E$ when $s \rightarrow t$.

Furthermore given fixed function $f: J \times E \rightarrow E$ we shall denote: for each $t \in J$ let

$$
\bar{f}_{t}(x):=f(t, x), \quad x \in E .
$$

Thus we have $\bar{f}_{t} \in E^{E}$ for $t \in J$.

Now we can formulate a theorem that gives (in terms of the function $\bar{f}_{t}$ ) necessary conditions for any space $E$ and sufficient ones when $\operatorname{dim} E<\infty$ such that superposition operator $F_{f}$ maps $R(J, E)$ into itself.

Theorem 4.4. Let the superposition operator $F_{f}$ maps $R(J, E)$ into itself. Then the family of functions $\left\{\bar{f}_{t}\right\}_{t \in J} \subset$ $E^{E}$ satisfies the following conditions:

(a) The mapping $J \ni t \mapsto \bar{f}_{t} \in E^{E}$ is a regulated function.

(b) The following limits of pointwise convergence exist and

(b1) $\lim _{s \rightarrow t^{+}} \bar{f}_{s}$ is continuous in $E$ for $t \in[a, b)$,

(b2) $\lim _{s \rightarrow t^{-}} \bar{f}_{s}$ is continuous in $E$ for $t \in(a, b]$.

Conversely, if additionally $E$ is a finitely dimensional Banach space and conditions (a) and (b) are satisfied then the superposition operator $F_{f}$ maps $R(J, E)$ into itself.

Proof. ( $\Rightarrow$ ) Let us fix $t \in[a, b)$. Using condition (2) of Theorem 4.1 and based on notation (4.1) we have the following equality

$$
g_{t}(x)=\lim _{s \rightarrow t^{+}} \bar{f}_{s}(x), \quad x \in E .
$$

First we prove (b1). Let us fix $x \in E$ and $\varepsilon>0$. Because of (4.2) we have the existence of $\delta>0$ and $\tau>0$ such that

$$
\left\|g_{t}(x)-\bar{f}_{s}(v)\right\| \leq \varepsilon, \quad s \in(t, t+\tau), v \in B(x, \delta) .
$$

Now going from $s \rightarrow t^{+}$we have $\left\|g_{t}(x)-g_{t}(v)\right\| \leq \varepsilon$ which proves continuity of $g_{t}$ in $x$ and thereby on $E$. Analogically we can prove (b2). 
We will now prove (a) i.e. that $\bar{f}_{s}$ converges to $g_{t}$ almost uniformly on $E$ when $s \rightarrow t^{+}$. Let us fix non-empty and compact set $K \subset E$ and $\varepsilon>0$. Then, because of (4.2) and already proven continuity of $g_{t}$, we have that for each $x \in K$ there exist $\delta_{x}>0, \tau_{x}>0$ such that concurrently

$$
\forall_{s \in\left(t, t+\tau_{x}\right)} \forall_{v \in B_{E}\left(x, \delta_{x}\right)}\left\|g_{t}(x)-\bar{f}_{s}(v)\right\| \leq \frac{\varepsilon}{2}
$$

and

$$
\left\|g_{t}(x)-g_{t}(v)\right\| \leq \frac{\varepsilon}{2}, \quad v \in B_{E}\left(x, \delta_{x}\right) .
$$

Out of family $\left\{B_{E}\left(x, \delta_{x}\right)\right\}_{x \in K}$ covering compact set $K$ we choose a finite subcover $\left\{B_{E}\left(x_{i}, \delta_{x_{i}}\right)\right\}_{i=1}^{n}$. Let $\bar{\tau}:=\min \left\{\tau_{i}: i=1, \ldots, n\right\}$. Let us fix arbitrary $v \in K$. Then there exists such $i$ that $v \in B_{E}\left(x_{i}, \delta_{i}\right)$. Thus for $s \in(t, t+\bar{\tau})$ based on (4.3) and (4.4) we have the following estimation

$$
\left\|g_{t}(v)-\bar{f}_{s}(v)\right\| \leq\left\|g_{t}(v)-g_{t}\left(x_{i}\right)\right\|+\left\|g_{t}\left(x_{i}\right)-\bar{f}_{s}(v)\right\| \leq \frac{\varepsilon}{2}+\frac{\varepsilon}{2}=\varepsilon
$$

for any $v \in K$, i.e. we have uniform convergence on $K$. Similarly we can prove the existence of the $\operatorname{limit}_{s \rightarrow t^{-}} \lim _{s}$ in the topology of almost uniform convergence.

$(\Leftarrow)$ Assume that $E$ has a finite dimension and fix $t \in[a, b)$. Condition (a) assures the existence of the limit $g_{t}:=\lim _{s \rightarrow t^{+}} \bar{f}_{s}$ which, based on (b) is continuous on $E$. Let us fix $x \in E$ and $\varepsilon>0$. Continuity of $g_{t}$ means that for some $r>0$ we have

$$
\left\|g_{t}(x)-g_{t}(v)\right\| \leq \frac{\varepsilon}{2}, \quad v \in B_{E}(x, r) .
$$

Moreover (a) implies that for a compact set $\bar{B}_{E}(x, r)$ there exists $\tau>0$ such that

$$
\left\|g_{t}(v)-\bar{f}_{s}(v)\right\| \leq \frac{\varepsilon}{2}, \quad v \in \bar{B}_{E}(x, r), s \in(t, t+\tau) .
$$

When we combine it with (4.5), for $v \in B_{E}(x, r), s \in(t, t+\tau)$ we have

$$
\left\|g_{t}(x)-f(s, v)\right\| \leq\left\|g_{t}(x)-g_{t}(v)\right\|+\left\|g_{t}(x)-f(s, v)\right\| \leq \frac{\varepsilon}{2}+\frac{\varepsilon}{2}=\varepsilon
$$

i.e. condition (4.2) is satisfied and thereby (2) in Theorem 4.1 holds. Similarly we can prove (1) in Theorem 4.1, so actually $F_{f}$ acts from $R(J, E)$ into $R(J, E)$.

Corollary 4.5. If $E$ is a finitely dimensional Banach space then superposition operator $F_{f}$ acts from $R(J, E)$ into $R(J, E)$ if and only if both conditions (a) and (b) in Theorem 4.4 are satisfied.

Now we give further sufficient and necessary conditions that superposition operator $F_{f}$ acts from $R(J, E)$ into $R(J, E)$.

To this end, let us recall so-called module of continuity of a mapping $h: E \rightarrow E$ at a point $v \in E$ given by

$$
v(h, v, \delta):=\sup \left\{\|h(v)-h(u)\|: u \in B_{E}(v, \delta)\right\}, \delta>0 .
$$

For a fixed mapping $f: J \times E \rightarrow E$ and $v \in E$ let us denote

$$
D_{v}:=\left\{t \in J \text { : the mapping } \bar{f}_{t} \text { is not continuous at } v\right\} .
$$

For any subset $S \subset J$, in the space $R(J, E)$ we will use a pseudonorm $\|\cdot\|_{S}$ given by

$$
\|x\|_{S}:=\sup \{\|x(t)\|: t \in S\}, \quad x \in R(J, E) .
$$

Now we can give another sufficient and necessary criterion that superposition operator $F_{f}$ acts from $R(J, E)$ into $R(J, E)$.

Theorem 4.6. Superposition operator $F_{f}$ acts from $R(J, E)$ into $R(J, E)$ if and only if the following four conditions are satisfied:

(a) $\forall_{v \in E} f(\cdot, v) \in R(J, E)$. 
(b) For each $v \in E$ set $D_{v}$ is finite or countable.

(c) For each $v \in E$, if the set $D_{v}=\left\{t_{n}\right\}$ is infinite then

$$
\lim _{(n, \delta) \rightarrow\left(\infty, 0^{+}\right)} v\left(\bar{f}_{t_{n}}, v, \delta\right)=0 .
$$

(d) The mapping $E \ni u \mapsto f(\cdot, u) \in R(J, E)$ is continuous in every point $v \in E$ in regard to pseudonorm $\|\cdot\|_{J \backslash D_{v}}$, i.e. for each $v \in E$ and each sequence $v_{n} \rightarrow v$ we have $\left\|f(\cdot, v)-f\left(\cdot, v_{n}\right)\right\|_{J \backslash D_{v}} \rightarrow 0$ when $n \rightarrow \infty$.

Before we prove Theorem 4.6 we give technical lemma, necessary in the next part of the paper.

Lemma 4.7. If there exist a number $\varepsilon_{0}>0$, a sequence $\left\{t_{n}\right\} \subset J$ convergent to a point $t_{0} \in J$ from one side and a sequence $\left\{v_{n}\right\} \subset E$ convergent to vector $v \in E$, such that

$$
\left\|f\left(t_{n}, v\right)-f\left(t_{n}, v_{n}\right)\right\| \geq \varepsilon_{0}, \quad n=1,2, \ldots
$$

then superposition operator $F_{f}$ does not act from $R(J, E)$ into $R(J, E)$.

Proof. We give a proof by contradiction. Let us assume that $F_{f}$ acts from $R(J, E)$ into $R(J, E)$. Hence we have

$$
\forall v \in E(\cdot, v) \in R(J, E) .
$$

Let assume that $\left\{t_{n}\right\}$ is convergent to some $t_{0} \in J$ from one side, for example $t_{n} \rightarrow t_{0}^{+}$, and moreover $\left\{t_{n}\right\}$ is strictly decreasing (we can have that choosing a proper subsequence). Let us put

$$
y(t):= \begin{cases}\theta & \text { for } t \in\left[a, t_{0}\right] \cup\left(t_{1}, b\right] \\ v_{n} & \text { for } t \in\left(t_{n+1}, t_{n}\right], n=1,2, \ldots .\end{cases}
$$

Obviously $y \in R(J, E)$. Since $F_{f} y \in R(J, E)$, then there exists a limit

$$
\lim _{t \rightarrow t_{0}^{+}}\left(F_{f} y\right)(t)=\lim _{n \rightarrow \infty} f\left(t_{n}, v_{n}\right) .
$$

Moreover, by (4.9), there exists a limit $\lim _{n \rightarrow \infty} f\left(t_{n}, v\right)$. Hence, by (4.8) we get

$$
\left\|\lim _{n \rightarrow \infty} f\left(t_{n}, v_{n}\right)-\lim _{n \rightarrow \infty} f\left(t_{n}, v\right)\right\| \geq \varepsilon_{0} .
$$

Now let us define next function $z \in R(J, E)$ as follows

$$
z(t):= \begin{cases}\theta & \text { for } t \in\left[a, t_{0}\right] \cup\left(t_{1}, b\right], \\ v & \text { for } t \in\left(t_{2 n}, t_{2 n-1}\right], n=1,2, \ldots, \\ v_{n} & \text { for } t \in\left(t_{2 n+1}, t_{2 n}\right], n=1,2, \ldots .\end{cases}
$$

Since $F_{f} z \in R(J, E)$ then there is a limit $\lim _{t \rightarrow t_{0}^{+}}\left(F_{f} z\right)(t)$ which means that

$$
\lim _{n \rightarrow \infty} f\left(t_{2 n}, v_{2 n}\right)=\lim _{n \rightarrow \infty} f\left(t_{2 n-1}, v\right) .
$$

However it is in contradiction with (4.10).

Proof of Theorem $4.6(\Rightarrow)$ Take an arbitrary $v \in E$ and put $x(t) \equiv v, t \in J$. Since $F_{f} x \in R(J, E)$, then (a) is satisfied.

Let us assume that the set $D_{v}$ is uncountable. Since

$$
D_{v}=\bigcup_{k=1}^{\infty}\left\{t \in J: v\left(\bar{f}_{t}, v\right)>\frac{1}{k}\right\}
$$

then there exists such $k \in \mathbb{N}$, that the set $\left\{t \in J: v\left(\bar{f}_{t}, v\right)>\frac{1}{k}\right\}$ is uncountable. Let us put $\varepsilon_{0}:=\frac{1}{k}$ and choose an arbitrary injective sequence $\left\{t_{n}\right\} \subset\left\{t \in J: v\left(\bar{f}_{t}, v\right)>\frac{1}{k}\right\}$. Since $v\left(\bar{f}_{t_{n}}, v\right)>\varepsilon_{0}$ for $n=1,2, \ldots$ it follows 
that for each $n \in \mathbb{N}$ we can choose such $v_{n} \in E$, that $\left\|\bar{f}_{t_{n}}(v)-\bar{f}_{t_{n}}\left(v_{n}\right)\right\| \geq \varepsilon_{0}$ and $\left\|v-v_{n}\right\| \leq \frac{1}{n}$. Choosing from the sequence $\left\{t_{n}\right\}$ a subsequent (also denoted as $\left\{t_{n}\right\}$ ) which converges from one side to some $t \in J$ we have $v_{n} \rightarrow v$ and

$$
\left\|f\left(t_{n}, v\right)-f\left(t_{n}, v_{n}\right)\right\| \geq \varepsilon_{0}, \quad n=1,2, \ldots .
$$

However then, by Lemma 4.7 operator $F_{f}$ would not act from $R(J, E)$ into $R(J, E)$ which proves (b).

Analogically negating conditions (c) and (d) we would have the existence of $\varepsilon_{0}>0$, a sequence $\left\{t_{n}\right\} \subset D_{v}$ for condition (c) or $\left\{t_{n}\right\} \subset J \backslash D_{v}$ for condition (d), convergent from one side to some $t \in J$, a vector $v \in E$ and a sequence $\left\{v_{n}\right\} \subset E$, such that $v_{n} \rightarrow v$ and (4.11) holds which, by Lemma 4.7 contradicts the hypothesis and thereby prove conditions (c) and (d).

$(\Leftarrow)$ Let us fix $x \in R(J, E)$. To prove that $F_{f} x \in R(J, E)$ we will show that for fixed $t \in[a, b)$ there exists a limit $\lim _{s \rightarrow t^{+}}\left(F_{f} x\right)(s)$ (we omit the proof of the existence of left-hand side limit as similar to the following). Let us fix such a sequence $\left\{t_{n}\right\} \subset(t, b]$, that $t_{n} \rightarrow t^{+}$and define $v_{n}:=x\left(t_{n}\right)$. Since $x \in R(J, E)$, then there exists a limit $v:=\lim _{n \rightarrow \infty} v_{n}$. By (a) there exists also a limit $\lim _{n \rightarrow \infty} f\left(t_{n}, v\right)$. To prove the existence of the $\operatorname{limit}_{s \rightarrow t^{+}}\left(F_{f} x\right)(s)$ it is enough to show the existence of the $\operatorname{limit}_{n \rightarrow \infty} \lim _{n \rightarrow \infty}\left(t_{n}, v_{n}\right)$. Additionally we show that

$$
\lim _{n \rightarrow \infty} f\left(t_{n}, v_{n}\right)=\lim _{n \rightarrow \infty} f\left(t_{n}, v\right) .
$$

Let us consider 3 cases:

Case (i): the sequence $\left\{t_{n}\right\}$, outside a finite number of terms is contained in $J \backslash D_{v}$.

Therefore, by (d), for each $\varepsilon>0$ and for sufficiently large $n$ we have $\left\|f\left(t_{n}, v_{n}\right)-f\left(t_{n}, v\right)\right\| \leq \varepsilon$. When $n \rightarrow \infty$ we have

$$
\limsup _{n \rightarrow \infty}\left\|f\left(t_{n}, v_{n}\right)-\lim _{n \rightarrow \infty} f\left(t_{n}, v\right)\right\| \leq \varepsilon
$$

which considering that $\varepsilon>0$ is arbitrary implies (4.12).

Case (ii): The sequence $\left\{t_{n}\right\}$, outside a finite number of terms is contained in $D_{v}$.

Let us fix $\varepsilon>0$. By (b) and (c) there exists such $n_{0} \in \mathbb{N}$ and $\delta_{0}>0$, that for $n \geq n_{0}$ and for $0<\delta<\delta_{0}$ we have $v\left(\bar{f}_{t_{n}}, v, \delta\right)<\varepsilon$, i.e. for large enough $n$ we have $\left\|f\left(t_{n}, v_{n}\right)-f\left(t_{n}, v\right)\right\| \leq \varepsilon$. When $n \rightarrow \infty$ using similar reasoning as in case (i) we also get that (4.12) holds.

Case (iii): Infinitely many terms of the sequence $\left\{t_{n}\right\}$ is contained in $D_{v}$ as well as infinitely many terms of the sequence $\left\{t_{n}\right\}$ is contained in $J \backslash D_{v}$.

Dividing the sequence $\left\{t_{n}\right\}$ into two adequate subsequences the case can be reduced to previous cases (i) and (ii).

Remark 4.8. The above Theorems 4.4 and 4.6 improve some mistake contained in [13] (Theorem 5.1).

We now give a criterion of compactness for the superposition operator $F_{f}$.

Theorem 4.9. Let $E$ be a separable Banach space and let the superposition operator $F_{f}: R(J, E) \rightarrow R(J, E)$ be continuous and compact (i.e. $F_{f}$ transforms bounded sets in relatively compact sets). Then

(H1) there exists a function $g \in R(J, E)$,

(H2) there exists a countable or finite set $T=\left\{t_{n}\right\} \subset J$,

(H3) there exist a countable or finite sequence of functions $h_{n}: E \rightarrow E, n=1,2, \ldots$ that are continuous, compact and when it is infinite

$$
\forall r>0 \lim _{n \rightarrow \infty}\left\|h_{n}\left(B_{E}(r)\right)\right\|=0
$$

such that

$$
f(t, x)=g(t)+\sum_{n=1}^{\infty} \mathbf{1}_{t_{n}}(t) h_{n}(x), \quad t \in J, x \in E .
$$

Conversely, if the conditions (H1)-(H3) are satisfied and $E$ is a Banach space then the formula (4.14) gives such a function $f(t, x)$, that operator $F_{f}: R(J, E) \rightarrow R(J, E)$ and it is continuous and compact.

Remark 4.10. Obviously the case when all $h_{n}$ functions in the previous theorem are equal to $\theta$, that is when $f(t, x)=g(t)$, or only a finite number of them is not equal to $\theta$ is also allowed. 
The proof of the theorem will be preceded by two lemmas. Before that however we will give a useful notation. For each $x \in R(J, E)$ we will put

$$
\operatorname{supp} x:=\{t \in J: x(t) \neq \theta\} .
$$

In contrast to standard definition of a support we do not require the closure.

Lemma 4.11. If $F_{f}: R(J, E) \rightarrow R(J, E)$ is compact, then for each $x, y \in R(J, E)$ the $\operatorname{set} \operatorname{supp}\left(F_{f} x-F_{f} y\right)$ is countable or finite and for each injective sequence $\left\{t_{n}\right\} \subset J$, we have

$$
\lim _{n \rightarrow \infty} f\left(t_{n}, x\left(t_{n}\right)\right)-f\left(t_{n}, y\left(t_{n}\right)\right)=\theta .
$$

Proof. If the set $\operatorname{supp}\left(F_{f} x-F_{f} y\right)$ was uncountable or if (4.15) was not satisfied, then there would exist a injective sequence $\left\{s_{n}\right\} \subset J$ and a number $\varepsilon_{0}>0$, such that

$$
\left\|f\left(s_{n}, x\left(s_{n}\right)\right)-f\left(s_{n}, y\left(s_{n}\right)\right)\right\| \geq \varepsilon_{0}, \quad n=1,2, \ldots
$$

Let us define the sequence of functions $u_{n}: J \rightarrow E, n=1,2, \ldots$ by

$$
u_{n}(t):= \begin{cases}x\left(s_{n}\right) & \text { for } t=s_{n}, \\ y(t) & \text { for } t \neq s_{n} .\end{cases}
$$

Obviously $u_{n} \in R(J, E)$. For $n \neq m$ we have $s_{n} \neq s_{m}$ and therefore

$$
\begin{gathered}
\left\|F_{f} u_{n}-F_{f} u_{m}\right\|_{\infty} \geq\left\|\left(F_{f} u_{n}\right)\left(s_{n}\right)-\left(F_{f} u_{m}\right)\left(s_{n}\right)\right\| \\
=\left\|f\left(s_{n}, x\left(s_{n}\right)\right)-f\left(s_{n}, y\left(s_{n}\right)\right)\right\| \geq \varepsilon_{0}
\end{gathered}
$$

i.e. the sequence $\left\{F_{f} u_{n}\right\}$ is $\varepsilon_{0}$-separable, hence $F_{f}$ is not compact, which ends the proof.

Lemma 4.12. If $E$ is a separable Banach space and $F_{f}: R(J, E) \rightarrow R(J, E)$ is compact and continuous, then there exists a countable or finite set $T=\left\{t_{n}\right\} \subset J$, such that

$$
\forall_{x \in E} \operatorname{supp}\left(F_{f} \widehat{x}-F_{f} \widehat{\theta}\right) \subset T \text {. }
$$

Proof. Let $A=\left\{a_{n}: n \in \mathbb{N}\right\} \subset E$ be a countable dense subset of $E$. Let us put $T:=\cup_{n=1}^{\infty} \operatorname{supp}\left(F_{f} \widehat{a_{n}}-F_{f} \widehat{\theta}\right)$. By the previous Lemma 4.11, the set $T$ is countable or finite. If there existed $x \in E$ such that (4.16) did not hold then there would exist $s \in J$ such that $s \in \operatorname{supp}\left(F_{f} \widehat{x}-F_{f} \widehat{\theta}\right) \backslash T$. Thus $\left\|\left(F_{f} \widehat{x}-F_{f} \widehat{\theta}\right)(s)\right\|=\varepsilon_{0}$ for some $\varepsilon_{0}>0$ and additionally $\left(F_{f} \widehat{a_{n}}-F_{f} \widehat{\theta}\right)(s)=\theta$ for $n \in \mathbb{N}$. If we took such a subsequence $\left\{a_{k_{n}}\right\}$, that $a_{k_{n}} \rightarrow x$ in $E$ we would have

$$
\left\|F_{f} \widehat{x}-F_{f} \widehat{a_{k_{n}}}\right\|_{\infty} \geq\left\|\left(F_{f} \widehat{x}-F_{f} \widehat{\theta}\right)(s)-\left(F_{f} \widehat{a_{k_{n}}}-F_{f} \widehat{\theta}\right)(s)\right\|=\varepsilon_{0}
$$

which is in contradiction with continuity $F_{f}$.

Proof. (Proof of Theorem 4.9)

Let us assume first that $E$ is separable and $F_{f}$ is compact and continuous. Let the set $T=\left\{t_{n}\right\}$ be like in Lemma 4.12. We define the function $g: J \rightarrow E$ and the sequence of functions $h_{n}: E \rightarrow E, n=1,2, \ldots$ by

$$
\begin{gathered}
g(t):=f(t, \theta), \quad t \in J, \\
h_{n}(x):=f\left(t_{n}, x\right)-f\left(t_{n}, \theta\right), \quad x \in E, n \in \mathbb{N} .
\end{gathered}
$$

By Lemma 4.12, for each $x \in E$ the mapping $J \ni t \rightarrow f(t, x)-f(t, \theta)$ can be non-zero only on the set $T$ and its formula is given by $\sum_{n=1}^{\infty} \mathbf{1}_{t_{n}}(t) h_{n}(x)$. Therefore

$$
f(t, x)=f(t, \theta)+f(t, x)-f(t, \theta)=g(t)+\sum_{n=1}^{\infty} \mathbf{1}_{t_{n}}(t) h_{n}(x) .
$$


Since $F_{f}$ is continuous the functions $h_{n}$ must be continuous and since $F_{f}$ is compact also the functions $h_{n}$ must be compact. Moreover it results from Lemma 4.11 that $\lim _{n \rightarrow \infty} f\left(t_{n}, x\right)-f\left(t_{n}, \theta\right)=\theta$ for $x \in E$ i.e.

$$
\lim _{n \rightarrow \infty} h_{n}(x)=\theta, \quad x \in E .
$$

We have only (4.13) left to prove. Let us assume that it is not satisfied. Then there would exist $r>0$, a number $\varepsilon_{0}>0$ and such a subsequence of the sequence $\left\{h_{n}\right\}$, (also denoted by $\left\{h_{n}\right\}$ ), that

$$
\left\|h_{n}\left(B_{E}(r)\right)\right\| \geq \varepsilon_{0}, \quad n=1,2, \ldots
$$

Let $k_{1}=1$. We choose $x_{1} \in B_{E}(r)$, such that $\left\|h_{k_{1}}\left(x_{1}\right)\right\| \geq \frac{\varepsilon_{0}}{2}$. By (4.17) we know that there exists $k_{2} \in \mathbb{N}$, such that $k_{2}>k_{1} \mathrm{i}\left\|h_{i}\left(x_{1}\right)\right\| \leq \frac{\varepsilon_{0}}{4}$ for $i \geq k_{2}$. By (4.18) we conclude that there exists $x_{2} \in B_{E}(r)$, such that $\left\|h_{k_{2}}\left(x_{2}\right)\right\| \geq \frac{\varepsilon_{0}}{2}$. By (4.17) we know that there exists $k_{3} \in \mathbb{N}$, such that $k_{3}>k_{2}$ and $\left\|h_{i}\left(x_{2}\right)\right\| \leq \frac{\varepsilon_{0}}{4}$ for $i \geq k_{3}$. Continuing this procedure we get an strictly increasing sequence $\left\{k_{n}\right\} \subset \mathbb{N}$ and the sequence $\left\{x_{n}\right\} \subset B_{E}(r)$ such that

$$
\begin{gathered}
\left\|h_{k_{n}}\left(x_{n}\right)\right\| \geq \frac{\varepsilon_{0}}{2}, \quad n=1,2, \ldots \\
\left\|h_{i}\left(x_{n}\right)\right\| \leq \frac{\varepsilon_{0}}{4}, \quad i \geq k_{n+1}, n=1,2, \ldots
\end{gathered}
$$

Let $n>m$. Then we have

$$
\begin{gathered}
\left\|F_{f} \widehat{x_{n}}-F_{f} \widehat{x_{m}}\right\|_{\infty} \geq\left\|\left(F_{f} \widehat{x_{n}}\right)\left(t_{k_{n}}\right)-\left(F_{f} \widehat{x_{m}}\right)\left(t_{k_{n}}\right)\right\| \\
=\left\|h_{k_{n}}\left(x_{n}\right)-h_{k_{n}}\left(x_{m}\right)\right\| \geq\left\|h_{k_{n}}\left(x_{n}\right)\right\|-\left\|h_{k_{n}}\left(x_{m}\right)\right\| \geq \frac{\varepsilon_{0}}{2}-\frac{\varepsilon_{0}}{4}=\frac{\varepsilon_{0}}{4}
\end{gathered}
$$

i.e. $\left\{F_{f} \widehat{x_{n}}\right\}$ is positively separated and $F_{f}$ is not compact which contradicts the assumptions.

Let us assume now that the conditions (H1)-(H3) are satisfied and the function $f(t, x)$ is given by the formula (4.14). First we prove that $F_{f}: R(J, E) \rightarrow R(J, E)$. Let us fix $x \in R(J, E), t \in J$ and the sequence $\left\{t_{j}^{\prime}\right\}$ convergent to $t$ from one side, e.g. $t_{j}^{\prime} \rightarrow t^{+}$. Since the sequence $\left\{x\left(t_{j}^{\prime}\right)\right\}$ is bounded, then by (4.13) we have $\lim _{j \rightarrow \infty} \sum_{n=1}^{\infty} \mathbf{1}_{t_{n}}\left(t_{j}^{\prime}\right) h_{n}\left(x\left(t_{j}^{\prime}\right)\right)=\theta$ and in virtue of (4.14) we get $\lim _{j \rightarrow \infty}\left(F_{f} x\right)\left(t_{j}^{\prime}\right)=\lim _{j \rightarrow \infty} g\left(t_{j}^{\prime}\right)=g\left(t^{+}\right)$i.e. $F_{f} x \in R(J, E)$.

Now we prove that the operator $F_{f}$ is compact. Let us fix a bounded sequence $\left\{x_{n}\right\} \subset B_{R(J, E)}(r)$, where $r>0$. Since the operator $h_{1}$ is compact we are able to choose such a subsequence $\left\{x_{n, 1}\right\}$ of a sequence $\left\{x_{n}\right\}$ that the sequence $\left\{h_{1}\left(x_{n, 1}\left(t_{1}\right)\right)\right\}$ is convergent. Since the operator $h_{2}$ is compact we are able to choose such a subsequence $\left\{x_{n, 2}\right\}$ of the sequence $\left\{x_{n, 1}\right\}$ that the sequence $\left\{h_{2}\left(x_{n, 2}\left(t_{2}\right)\right)\right\}$ is convergent. Obviously $\left\{h_{1}\left(x_{n, 2}\left(t_{1}\right)\right)\right\}$ is also convergent. Continuing this procedure we get a sequence of sequences $\left\{x_{n, i}\right\}_{n=1}^{\infty}, i=$ $1,2, \ldots$ which has a property that $\left\{x_{n, i+1}\right\}_{n=1}^{\infty}$ is a subsequence of the sequence $\left\{x_{n, i}\right\}_{n=1}^{\infty}$ and that there exists a limit $\lim _{n \rightarrow \infty} h_{j}\left(x_{n, i}\left(t_{j}\right)\right)$ for $i=1,2, \ldots, j=1,2, \ldots, i$. Now, using a diagonal method we can define the sequence $\left\{y_{n}\right\}$ by $y_{n}:=x_{n, n}, n=1,2, \ldots$ Obviously $\left\{y_{n}\right\}$ is a subsequence of $\left\{x_{n}\right\}$. Moreover the $\operatorname{limit}_{n \rightarrow \infty} \lim _{i}\left(y_{n}\left(t_{i}\right)\right)$ exists for each $i=1,2, \ldots$. Thus as well as from the fact that $\left(F_{f} y_{n}\right)(t)=g(t)$ for $t \notin T$ we conclude that the limit $\lim _{n \rightarrow \infty}\left(F_{f} y_{n}\right)(t)$ exists for each $t \in J$. We are able now to define a function $z: J \rightarrow E$ by $z(t):=\lim _{n \rightarrow \infty}\left(F_{f} y_{n}\right)(t)$ i.e.

$$
z(t)= \begin{cases}g(t) & \text { for } t \in J \backslash T, \\ g\left(t_{i}\right)+\lim _{n \rightarrow \infty} h_{i}\left(y_{n}\left(t_{i}\right)\right) & \text { for } t=t_{i}, i=1,2, \ldots\end{cases}
$$

By (4.13) we have

$$
\left\|z\left(t_{i}\right)-g\left(t_{i}\right)\right\|=\left\|\lim _{n \rightarrow \infty} h_{i}\left(y_{n}\left(t_{i}\right)\right)\right\| \leq\left\|h_{i}\left(B_{E}(r)\right)\right\| \underset{i \rightarrow \infty}{\longrightarrow} 0 .
$$

This means that $z \in R(J, E)$. Using (4.14) we get

$$
\left\|z-F_{f} y_{n}\right\|_{\infty}=\sup _{t_{i} \in T}\left\|\lim _{n \rightarrow \infty} h_{i}\left(y_{n}\left(t_{i}\right)\right)-h_{i}\left(y_{n}\left(t_{i}\right)\right)\right\| .
$$

Let us fix $\varepsilon>0$. By (4.13) there exists $m \in \mathbb{N}$ such that for $i>m$ we have $\left\|h_{i}\left(B_{E}(r)\right)\right\| \leq \varepsilon$ and therefore $\sup _{i>m}\left\|\lim _{n \rightarrow \infty} h_{i}\left(y_{n}\left(t_{i}\right)\right)-h_{i}\left(y_{n}\left(t_{i}\right)\right)\right\| \leq 2 \varepsilon$. Since there exists limit $\lim _{n \rightarrow \infty} h_{i}\left(y_{n}\left(t_{i}\right)\right), i=1,2, \ldots$, then there is $n_{0} \in \mathbb{N}$ such that for $n \geq n_{0}$ and for $i=1,2, \ldots, m$ we have

$$
\left\|\lim _{n \rightarrow \infty} h_{i}\left(y_{n}\left(t_{i}\right)\right)-h_{i}\left(y_{n}\left(t_{i}\right)\right)\right\| \leq \varepsilon .
$$


Combining the above and (4.19) we get for $n \geq n_{0}$ the inequality $\left\|z-F_{f} y_{n}\right\|_{\infty} \leq 2 \varepsilon$ which proves that $F_{f} y_{n} \rightarrow z$ in $R(J, E)$.

Continuity of the operator $F_{f}$ is a consequence of condition (H3) - we omit a simple proof of this fact.

Corollary 4.13. Let Banach space $E$ be separable. Then $F_{f}(R(J, E)) \subset R(J, E)$ and the superposition operator $F_{f}: R(J, E) \rightarrow R(J, E)$ is continuous and compact if and only if the conditions (H1)-(H3) of Theorem 4.9 are satisfied.

Problem 4.14. Are the conditions (H1)-(H3) of Theorem 4.9 necessary in case when the space $E$ is not separable?

\section{References}

[1] G. Aumann, Reelle Funktionen, Springer, Berlin, 1954.

[2] W. Aziz, N. Merentes and J.L. Sanchez, A note on the composition of regular functions, Z. Anal. Anwend. 33 (2014), 119-123.

[3] J. Banaś, T. Zając, On a measure of noncompactness in the space of regulated functions and its applications, Adv. Nonlinear Anal. 8 (2019), 1099-1110.

[4] K. Cichoń, M. Cichoń and M.M.A. Metwali, On some parameters in the space of regulated functions and their applications, Carpathian J. Math. 34 (2018), no. 1, 17-30.

[5] K. Cichoń, M. Cichoń and B. Satco, On regulated functions, Fasc. Math. 60 (2018) (in press).

[6] K. Cichoń, M. Cichoń and B. Satco, Measure Differential Inclusions Through Selection Principles in the Space of Regulated Functions, Mediterr. J. Math. (2018) 15:148 https://doi.org/10.1007/s00009-018-1192-y 1660-5446/18/040001-19

[7] J. Dieudonné, Foundations of Modern Analysis, Academic Press, New York, 1969.

[8] L. Drewnowski, On Banach spaces of regulated functions, Comment. Math. vol. 57, no. 2 (2017), 153-167.

[9] D. Fraňkova, Regulated functions, Math. Bohemica 116 (1991), 20-59.

[10] L.A.O. Fernandes and R. Arbach, Integral functionals on C-algebra of vector-valued regulated functions, Ann. Funct. Anal. 3(2012), 21-31.

[11] C. Goffman, G. Moran and Waterman, D., The structure of regulated functions, Proc. Amer. Math. Soc. 57 (1976), 61-65.

[12] A. Michalak, On superposition operators in spaces of regular and of bounded variation functions, Z. Anal. Anwend. 35 (2016), 285-308.

[13] L. Olszowy, Measures of noncompactness in the space of regulated functions, J. Math. Anal. Appl. 476 (2019), $860-874$.

[14] B. Satco, Regulated solutions for nonlinear measure driven equations, Nonlinear Anal. Hybrid Syst. 13 (2014), 22-31.

[15] J. Banaś and K. Goebel, Measures of Noncompactness in Banach Spaces, Lect. Notes in Pure and Appl. Math. 60, Marcel Dekker, New York, 1980.

[16] W. Orlicz and S. Szufla, On some classes of nolinear Volterra integral equations in Banach spaces, Bull. Pol. Acad. Sci. Math. 30 (1982) no 5-6, 239-250.

[17] H-P. Heinz, On the behaviour of measures of noncompactness with respect to differentiations and integration of vectorvalued functions, Nonlinear Anal. Vol 7 No 12 (1983), 1351-1371.

[18] P. Chen and Y. Li, Monotone iterative technique for a class of semilinear evolution equations with nonlocal conditions, Results Math. 63 (2013), 731-744.

[19] N. Ackermann, Uniform continuity and Brezis-Lieb-type splitting for superposition operators in Sobolev space, Adv. Nonlinear Anal. 7 (2018), no. 4, 587-599.

[20] J. Appell and P.P. Zabrejko, Nonlinear Superposition Operators, in: Cambridge Tracts in Mathematics, vol. 95, Cambridge University Press, 1990.

[21] N.S. Papageorgiou, V.D. Radulescu and D.D. Repovs, Nonlinear analysis - theory and methods, Springer Monographs in Mathematics, Springer, Berlin, 2019. 